\title{
Flows of incompressible viscous liquids with anisotropic wall slip
}

\author{
C. Le Roux \\ Department of Mathematics and Applied Mathematics, University of Pretoria, \\ Private Bag X20, Hatfield 0028, South Africa
}

\begin{abstract}
This paper deals with a boundary-value problem for the Stokes equations with a general direction-dependent Navier type slip boundary condition. This problem models the steady laminar flow of an incompressible linearly viscous liquid in a bounded domain with an impermeable rough boundary with variable and anisotropic roughness. It is proved that the problem has a unique weak solution.
\end{abstract}

Keywords: Stokes equations, slip boundary condition, rough boundary 2010 MSC: 76D03, 76D07

\section{Introduction}

During the past twenty years significant progress was made in the analysis of laminar flows of Newtonian liquids (e.g. water) over complex surfaces. The numerical simulation of such flows is greatly simplified if the complex boundary surface and microscopic boundary conditions are approximated by a smooth boundary and an effective boundary condition. Lee et al. [1] review physical experiments and computational studies conducted to deduce effective boundary conditions for flows over rough or structured surfaces, surfaces with chemical patterns, nano-bubbles or polymer layers, and superhydrophobic surfaces. In many of these situations, the proposed effective boundary condition is a partial slip boundary condition of the form introduced by Navier [2]. Lee et al. [1] discuss the progress and difficulties in deriving the effective Navier slip length in these situations.

During the same period several mathematical studies of flows over rough surfaces, involving various assumptions and techniques, yielded Navier type slip conditions as effective boundary conditions. These works deal with flows over one-dimensional boundaries with periodic irregularities, e.g. $[3,4,5,6]$, flows over two-dimensional boundaries with periodic irregularities, e.g. $[7,8,9,10$, $11,12]$, and flows over one-dimensional boundaries with random irregularities,

$U R L$ : ianleroux@yahoo.com (C. Le Roux) 
e.g. $[13,14,15]$. These are only a few examples; many other studies are reviewed in $[8,11,12,16]$.

When a rough or structured boundary surface is anisotropic, e.g. when it has rows of riblets, pillars or periodic patterns, the effective slip condition is anisotropic, i.e., direction-dependent. Examples of flows over such surfaces have been studied by analytical and numerical methods [16, 17, 18, 19]. However, these studies only consider flows in the directions perpendicular to and parallel to the rows of riblets. Moreover, to our knowledge, none of the works discussed by Lee et al. [1] or the mathematical studies mentioned above attempt to deduce a general anisotropic slip law. This is not surprising in view of the experimental difficulties and intricate mathematical analysis involved. (In Remark 1 an approach to derive such a slip law is suggested.) In addition, when the surface is heterogeneous, the effective slip is also heterogeneous, i.e., position-dependent. This can occur, for example, when the boundary has a varying degree of roughness or when the boundary is a smooth surface with a varying hydrophobic/hydrophilic composition.

The well-posedness of boundary-value problems for flows with position- and direction-dependent slip boundary conditions has apparently not been studied. The aim of the present work is to consider a boundary-value problem for the Stokes equations with a slip boundary condition that is in essence of the form

$$
(\mathbb{T} \boldsymbol{n})_{\tau}=-F\left(|\boldsymbol{v}|^{-1} \boldsymbol{v}\right) \boldsymbol{v} \text { on } \partial \Omega .
$$

Here $\partial \Omega$ is the impermeable boundary of the flow domain $\Omega \subset \mathbb{R}^{3}, \mathbb{T}$ is the Cauchy stress tensor, $\boldsymbol{n}$ is the outward unit normal vector, $(\mathbb{T} \boldsymbol{n})_{\tau}$ is the tangential component of the traction, $\boldsymbol{v}$ is the velocity, and the "friction coefficient" $F$ is a given function defined on

$$
S(\partial \Omega)=\left\{(x, \boldsymbol{u}) \in \partial \Omega \times \mathbb{R}^{3}: \boldsymbol{n}(x) \cdot \boldsymbol{u}=0,|\boldsymbol{u}|=1\right\} .
$$

Formally, condition (1) reduces to the condition of perfect (or free or frictionless) slip if $F \equiv 0$, and to the no-slip condition in the limit $F \longrightarrow \infty$. This is excluded here; it is assumed that $F$ takes values in some interval $\left[F_{L}, F_{U}\right]$ with $0<F_{L}<F_{U}$. This includes the case where $F$ is a positive constant, i.e., Navier's slip condition [2], and its generalization with $F=F(x)>0$. In the boundary-value problem considered here, $F$ is regularized to allow $|\boldsymbol{v}|=0$. This is done in Section 3. Then, in Section 4, we prove that the problem has a unique weak solution if the external body force is sufficiently small in comparison to the viscosity and the friction coefficient.

Remark 1. (a) We only consider the Stokes problem, but standard methods should suffice to extend the result to the corresponding Navier-Stokes problem.

(b) In our analysis it is not necessary to assume that $F(-\boldsymbol{u})=F(\boldsymbol{u})$. It is conceivable that $F(-\boldsymbol{u}) \neq F(\boldsymbol{u})$ for some $\boldsymbol{u}$ if condition (1) is used to approximate the apparent slip over a very rough surface with rows of unsymmetrical ridges.

(c) The effective slip conditions derived in the mathematical studies mentioned above are not always expressed as the familiar Navier condition. For 
example, in $[8,11]$ the tangential traction is formulated in terms of a matrix (called Navier's matrix) that is defined by boundary layer functions. Moreover, all these studies involve significant technical difficulties that require other techniques than those in this article.

(d) In a practical situation, an approximate slip condition of the type (1) could possibly be obtained as follows. Suppose for simplicity that the boundary surface is flat and covered by rows of pillars of varying heights. Then first consider the simpler situation where all the pillars have the same height. Use a suitable method (the computational method of [19], say) to derive effective Navier slip conditions in the form

$$
(\mathbb{T} \boldsymbol{n})_{\tau}=-N(h, \boldsymbol{u}) \boldsymbol{v},
$$

where $h$ is the height of the pillars and $\boldsymbol{u}$ is the flow direction, for a sufficient number of values of $h$ and directions $\boldsymbol{u}$ to approximate the function $N$. Then, for the original problem, replace $h$ by a local average, $h(x)$, of pillar heights.

\section{Notation}

$\Omega$ is a bounded domain in $\mathbb{R}^{3}$ with boundary $\partial \Omega$ of class $C^{1,1}$. For $1 \leq q \leq \infty$, $L^{q}(\Omega)$ and $L^{q}(\partial \Omega)$ are the standard Lebesgue spaces, with norms $\|\cdot\|_{q}$ and $\|\cdot\|_{q, \partial \Omega}$, respectively. When $q=2$, the inner products of these spaces are denoted by $(\cdot, \cdot)$ and $(\cdot, \cdot)_{\partial \Omega}$, respectively, and the norms are denoted by $\|\cdot\|$ and $\|\cdot\|_{\partial \Omega}$, respectively. The norms (and inner products, when $q=2$ ) in $L^{q}(\Omega)^{3}$ and $L^{q}(\partial \Omega)^{3}$ are denoted by the same symbols as in the scalar case.

For $m=1,2, H^{m}(\Omega)=W^{m, 2}(\Omega)$ is the standard Sobolev space, with inner product $(\cdot, \cdot)_{m, 2}$ and norm $\|\cdot\|_{m, 2}$, and $H^{m-1 / 2}(\partial \Omega)$ is the corresponding space of traces, with norm $\|\cdot\|_{m-1 / 2,2, \partial \Omega}$ (see, e.g., [20]). The inner products and norms in $H^{m}(\Omega)^{3}$ and $H^{m-1 / 2}(\partial \Omega)^{3}$ are denoted by the same symbols as in the scalar case. Furthermore, $\boldsymbol{n}$ is the outward unit normal vector on $\partial \Omega$ and

$$
\begin{aligned}
& U=\left\{\boldsymbol{v} \in H^{1}(\Omega)^{3}: \boldsymbol{v} \cdot \boldsymbol{n}=0 \text { on } \partial \Omega\right\}, \\
& V=\{\boldsymbol{v} \in U: \operatorname{div} \boldsymbol{v}=0 \text { in } \Omega\},
\end{aligned}
$$

where the boundary condition in the definition of $U$ is defined in the sense of traces.

\section{Problem formulation}

Let $F: S(\partial \Omega) \rightarrow \mathbb{R}$ be a function with the following properties:

(a) For all $(x, \boldsymbol{u}) \in S(\partial \Omega), F(\cdot, \boldsymbol{u})$ is continuous at $x$ in the sense that there is an open ball $B(x, r(x))=\left\{y \in \mathbb{R}^{3}:|y-x|<r(x)\right\}$ such that the function $E(\cdot, \boldsymbol{u})$ defined by

$$
E(y, \boldsymbol{u})=F\left(y,|(\boldsymbol{n}(y) \times \boldsymbol{u}) \times \boldsymbol{n}(y)|^{-1}(\boldsymbol{n}(y) \times \boldsymbol{u}) \times \boldsymbol{n}(y)\right)
$$

is continuous on $\partial \Omega \cap B(x, r(x))$. 
(b) $F(x, \cdot)$ is uniformly Lipschitz continuous, i.e., there exists $M_{0}>0$ such that for all $(x, \boldsymbol{u}),(x, \boldsymbol{v}) \in S(\partial \Omega)$,

$$
|F(x, \boldsymbol{u})-F(x, \boldsymbol{v})| \leq M_{0}|\boldsymbol{u}-\boldsymbol{v}| .
$$

(c) There exist constants $0<F_{L}<F_{U}$ such that for all $(x, \boldsymbol{u}) \in S(\partial \Omega)$,

$$
F_{L} \leq F(x, \boldsymbol{u}) \leq F_{U} .
$$

For every $x \in \partial \Omega$, let $\tau(x)$ be a unit tangential vector at $x$ and define the average of $F$ at $x$ by

$$
F_{a}(x)=\frac{1}{2 \pi} \int_{0}^{2 \pi} F(x, \boldsymbol{\tau}(x) \cos \theta+\boldsymbol{n}(x) \times \boldsymbol{\tau}(x) \sin \theta) d \theta .
$$

Let $T(\partial \Omega)=\left\{(x, \boldsymbol{v}) \in \partial \Omega \times \mathbb{R}^{3}: \boldsymbol{n}(x) \cdot \boldsymbol{v}=0\right\}$ and define $G: T(\partial \Omega) \rightarrow \mathbb{R}$ by

$$
\begin{aligned}
& G(x, \mathbf{0})=F_{a}(x), x \in \partial \Omega \\
& G(x, \boldsymbol{v})=F\left(x,|\boldsymbol{v}|^{-1} \boldsymbol{v}\right),(x, \boldsymbol{v}) \in T(\partial \Omega), \boldsymbol{v} \neq \mathbf{0} .
\end{aligned}
$$

Fix $0<r_{0}<r_{1} \leq 1$ and let $\varphi:[0, \infty) \rightarrow \mathbb{R}$ be a smooth (at least $C^{1}$ ) monotone function such that $\varphi(r)=0$ for all $r \in\left[0, r_{0}\right]$ and $\varphi(r)=1$ for all $r \geq r_{1}$. Let $M_{1}=\max \left\{\varphi^{\prime}(r): r \in\left[r_{0}, r_{1}\right]\right\}$. Define a regularization, $\widetilde{G}$, of $G$ : for $(x, \boldsymbol{v}) \in T(\partial \Omega)$,

$$
\widetilde{G}(x, \boldsymbol{v})=F_{a}(x)+\varphi(|\boldsymbol{v}|)\left(G(x, \boldsymbol{v})-F_{a}(x)\right) .
$$

Then define $H: U \rightarrow L^{\infty}(\partial \Omega)$ by

$$
H(\boldsymbol{v})(x)=\widetilde{G}(x,(\gamma \boldsymbol{v})(x)), x \in \partial \Omega,
$$

where $\gamma$ denotes the trace operator. Now consider the following Stokes problem:

Problem 1. Find $(\boldsymbol{v}, p)$ such that

$$
\begin{array}{rlrl}
-\mu \triangle \boldsymbol{v}+\nabla p & =\boldsymbol{f} & & \text { in } \Omega, \\
\operatorname{div} \boldsymbol{v}=0 & & \text { in } \Omega, \\
\boldsymbol{v} \cdot \boldsymbol{n} & =0 & & \text { on } \partial \Omega, \\
(\mathbb{T} \boldsymbol{n})_{\tau}+H(\boldsymbol{v}) \boldsymbol{v} & =\boldsymbol{g} & & \text { on } \partial \Omega .
\end{array}
$$

Here $\boldsymbol{v}$ is the velocity, $p$ is the pressure, $\mu$ is the viscosity, $\boldsymbol{f}$ is the external body force per unit volume, $\mathbb{T}=-p \mathbb{I}+2 \mu \mathbb{D}(\boldsymbol{v})$ is the Cauchy stress tensor with $\mathbb{D}(\boldsymbol{v})=\frac{1}{2}\left(\nabla \boldsymbol{v}+(\nabla \boldsymbol{v})^{T}\right)$, and $(\mathbb{T} \boldsymbol{n})_{\tau}=\mathbb{T} \boldsymbol{n}-(\boldsymbol{n} \cdot \mathbb{T} \boldsymbol{n}) \boldsymbol{n}$. In condition (12), $\boldsymbol{g}$ is an applied tangential surface traction. However, it is not clear how this can be realized physically and one may assume that $\boldsymbol{g} \equiv \mathbf{0}$ in the slip model considered here. A nonzero $\boldsymbol{g}$ is allowed for the sake of generality and because it does not create any additional mathematical difficulty. 
Let $\boldsymbol{f} \in L^{2}(\Omega)^{3}$ and $\boldsymbol{g} \in H^{1 / 2}(\partial \Omega)^{3}$ with $\boldsymbol{g} \cdot \boldsymbol{n}=0$ on $\partial \Omega$, and suppose that $(\boldsymbol{v}, p) \in H^{2}(\Omega)^{3} \times H^{1}(\Omega)$ is a solution of Problem 1. Then, by equations (9) and $(10),-\operatorname{div} \mathbb{T}=\boldsymbol{f}$ in $\Omega$. Thus $(-\operatorname{div} \mathbb{T}, \boldsymbol{\psi})=(\boldsymbol{f}, \boldsymbol{\psi})$ for all $\boldsymbol{\psi} \in V$. By applying Green's formula, the properties of $\boldsymbol{\psi}$, the symmetry of $\mathbb{D}(\boldsymbol{v})$, and boundary conditions (11)-(12), one deduces that for all $\boldsymbol{\psi} \in V$,

$$
a(\boldsymbol{v}, \boldsymbol{\psi})+(H(\boldsymbol{v}) \boldsymbol{v}, \boldsymbol{\psi})_{\partial \Omega}=(\boldsymbol{f}, \boldsymbol{\psi})+(\boldsymbol{g}, \boldsymbol{\psi})_{\partial \Omega},
$$

where $a(\cdot, \cdot)$ is the bilinear form defined by

$$
a(\boldsymbol{v}, \boldsymbol{w})=2 \mu(\mathbb{D}(\boldsymbol{v}), \mathbb{D}(\boldsymbol{w})), \boldsymbol{v}, \boldsymbol{w} \in H^{1}(\Omega)^{3} .
$$

The right-hand side of equation (13) defines a bounded linear functional on $V$, which is a closed subspace of $H^{1}(\Omega)^{3}$ and thus a Hilbert space with the inner product $(\cdot, \cdot)_{1,2}$. Hence, by the Riesz representation theorem, there exists a unique $\boldsymbol{h} \in V$ such that $(\boldsymbol{f}, \boldsymbol{w})+(\boldsymbol{g}, \boldsymbol{w})_{\partial \Omega}=(\boldsymbol{h}, \boldsymbol{w})_{1,2}$ for all $\boldsymbol{w} \in V$. Hence, for a given $\boldsymbol{h} \in H^{1}(\Omega)^{3}$, one has the following weak form of Problem 1:

Problem 2. Find $\boldsymbol{v} \in V$ such that for all $\boldsymbol{\psi} \in V$,

$$
a(\boldsymbol{v}, \boldsymbol{\psi})+(H(\boldsymbol{v}) \boldsymbol{v}, \boldsymbol{\psi})_{\partial \Omega}=(\boldsymbol{h}, \boldsymbol{\psi})_{1,2} .
$$

\section{Existence and uniqueness of a solution}

Theorem 1. There exist positive constants $C=C(\Omega), K=K\left(\Omega, \mu, F_{L}\right)$ and $M_{2}=M_{2}\left(F_{U}-F_{L}, M_{0}, \varphi\right)$ such that if

$$
\|\boldsymbol{h}\|_{1,2}<\frac{K^{2}}{C(\Omega) M_{2}}
$$

then Problem 2 has a unique solution, $\boldsymbol{v} \in V$. Moreover,

$$
\|\boldsymbol{v}\|_{1,2} \leq K^{-1}\|\boldsymbol{h}\|_{1,2} .
$$

The proof relies on the following two results. Their proofs are given after the proof of Theorem 1.

Lemma 1. Let $\Omega$ be a bounded domain in $\mathbb{R}^{3}$ with Lipschitz continuous boundary $\partial \Omega$ and let $\Gamma \subset \partial \Omega$ with $|\Gamma|>0$. Then there exist positive constants $K_{1}$ and $K_{2}$, which depend at most on $\Omega$ and $\Gamma$, such that for all $A, B>0$ and all $\boldsymbol{v} \in H^{1}(\Omega)^{3}$,

$$
A\|\mathbb{D}(\boldsymbol{v})\|^{2}+B\|\boldsymbol{v}\|_{\Gamma}^{2} \geq K_{1} \min \left\{K_{2} A, B\right\}\|\boldsymbol{v}\|_{1,2}^{2} .
$$

Lemma 2. For all $(x, \boldsymbol{v}) \in T(\partial \Omega)$,

$$
F_{L} \leq \widetilde{G}(x, \boldsymbol{v}) \leq F_{U} .
$$

Furthermore, there exists a constant $M_{2}=M_{2}\left(F_{U}-F_{L}, M_{0}, \varphi\right)$ such that for all $(x, \boldsymbol{v})$ and $(x, \boldsymbol{w})$ in $T(\partial \Omega)$,

$$
|\widetilde{G}(x, \boldsymbol{v})-\widetilde{G}(x, \boldsymbol{w})| \leq M_{2}|\boldsymbol{v}-\boldsymbol{w}| .
$$


Proof of Theorem 1 Suppose that $\boldsymbol{v} \in V$ is a solution of Problem 2. Set $\boldsymbol{\psi}=\boldsymbol{v}$ in equation (14). Then, by inequalities (17) and (18) 1 ,

$$
\begin{aligned}
K_{1} \min \left\{2 K_{2} \mu, F_{L}\right\}\|\boldsymbol{v}\|_{1,2}^{2} & \leq a(\boldsymbol{v}, \boldsymbol{v})+F_{L}\|\boldsymbol{v}\|_{\partial \Omega}^{2} \\
& \leq a(\boldsymbol{v}, \boldsymbol{v})+(H(\boldsymbol{v}) \boldsymbol{v}, \boldsymbol{v})_{\partial \Omega}=(\boldsymbol{h}, \boldsymbol{v})_{1,2} \\
& \leq\|\boldsymbol{h}\|_{1,2}\|\boldsymbol{v}\|_{1,2}
\end{aligned}
$$

and thus

$$
\|\boldsymbol{v}\|_{1,2} \leq R:=K^{-1}\|\boldsymbol{h}\|_{1,2},
$$

where $K:=K_{1} \min \left\{2 K_{2} \mu, F_{L}\right\}$.

If $\boldsymbol{h} \equiv \mathbf{0}$ then $\boldsymbol{v} \equiv \mathbf{0}$ is a solution of Problem 2. Moreover, by estimate (21), it is the only solution.

If $R>0$, let $\boldsymbol{u} \in B(V, R):=\left\{\boldsymbol{v} \in V:\|\boldsymbol{v}\|_{1,2} \leq R\right\}$ and consider the following auxiliary problem: Find $\boldsymbol{v} \in V$ such that

$$
b(\boldsymbol{u} ; \boldsymbol{v}, \boldsymbol{\psi})=(\boldsymbol{h}, \boldsymbol{\psi})_{1,2} \text { for all } \boldsymbol{\psi} \in V,
$$

where $b(\boldsymbol{u} ; \cdot, \cdot)$ is the bilinear form defined by

$$
b(\boldsymbol{u} ; \boldsymbol{v}, \boldsymbol{w})=a(\boldsymbol{v}, \boldsymbol{w})+(H(\boldsymbol{u}) \boldsymbol{v}, \boldsymbol{w})_{\partial \Omega}, \boldsymbol{v}, \boldsymbol{w} \in H^{1}(\Omega)^{3} .
$$

By the Cauchy-Schwarz inequality, inequality $(18)_{2}$ and the trace theorem,

$$
|b(\boldsymbol{u} ; \boldsymbol{v}, \boldsymbol{w})| \leq 2 \mu\|\nabla \boldsymbol{v}\| \cdot\|\nabla \boldsymbol{w}\|+C(\Omega) F_{U}\|\boldsymbol{v}\|_{1,2}\|\boldsymbol{w}\|_{1,2}
$$

for all $\boldsymbol{v}, \boldsymbol{w} \in H^{1}(\Omega)^{3}$, i.e. the bilinear form $b(\boldsymbol{u} ; \cdot, \cdot)$ is continuous on $H^{1}(\Omega)^{3} \times$ $H^{1}(\Omega)^{3}$ and thus on $V \times V$. Furthermore, by inequalities (18) $)_{1}$ and (17),

$$
b(\boldsymbol{u} ; \boldsymbol{v}, \boldsymbol{v}) \geq K\|\boldsymbol{v}\|_{1,2}^{2} \text { for all } \boldsymbol{v} \in H^{1}(\Omega)^{3},
$$

i.e. $b(\boldsymbol{u} ; \cdot, \cdot)$ is $H^{1}(\Omega)^{3}$-elliptic and thus $V$-elliptic. Hence, by the Lax-Milgram theorem [21, Theorem 2.1], equation (22) has a unique solution $\boldsymbol{v} \in V$. Moreover, by the same argument as in (20)-(21), $\|\boldsymbol{v}\|_{1,2} \leq R$. Thus one can define a mapping $\Phi: B(V, R) \rightarrow B(V, R)$ by $\Phi(\boldsymbol{u})=\boldsymbol{v}$. Then, by estimate (21), $\boldsymbol{v}$ is a solution of Problem 2 if and only if it is a fixed point of $\Phi$.

Let $\boldsymbol{u}, \hat{\boldsymbol{u}} \in B(V, R)$ and let $\boldsymbol{v}=\Phi(\boldsymbol{u}), \hat{\boldsymbol{v}}=\Phi(\hat{\boldsymbol{u}})$. Subtract equation (22) with $\hat{\boldsymbol{u}}$ and $\hat{\boldsymbol{v}}$ from equation (22) with $\boldsymbol{u}$ and $\boldsymbol{v}$ and set $\boldsymbol{\psi}=\boldsymbol{v}-\hat{\boldsymbol{v}}$. This gives

$$
a(\boldsymbol{v}-\hat{\boldsymbol{v}}, \boldsymbol{v}-\hat{\boldsymbol{v}})+(H(\boldsymbol{u}) \boldsymbol{v}-H(\hat{\boldsymbol{u}}) \hat{\boldsymbol{v}}, \boldsymbol{v}-\hat{\boldsymbol{v}})_{\partial \Omega}=0 .
$$

Thus, by inequalities (17) and (18) ${ }_{1}$, Hölder's inequality, inequality (19) and the continuity of the trace operator $\gamma: H^{1}(\Omega) \rightarrow L^{4}(\partial \Omega)$ (see, e.g., [22, Theorem II.4.1]),

$$
\begin{aligned}
K\|\boldsymbol{v}-\hat{\boldsymbol{v}}\|_{1,2}^{2} & \leq a(\boldsymbol{v}-\hat{\boldsymbol{v}}, \boldsymbol{v}-\hat{\boldsymbol{v}})+F_{L}\|\boldsymbol{v}-\hat{\boldsymbol{v}}\|_{\partial \Omega}^{2} \\
& \leq a(\boldsymbol{v}-\hat{\boldsymbol{v}}, \boldsymbol{v}-\hat{\boldsymbol{v}})+(H(\boldsymbol{u})(\boldsymbol{v}-\hat{\boldsymbol{v}}), \boldsymbol{v}-\hat{\boldsymbol{v}})_{\partial \Omega} \\
& =((H(\hat{\boldsymbol{u}})-H(\boldsymbol{u})) \hat{\boldsymbol{v}}, \boldsymbol{v}-\hat{\boldsymbol{v}})_{\partial \Omega} \\
& \leq\|H(\hat{\boldsymbol{u}})-H(\boldsymbol{u})\|_{\partial \Omega}\|\hat{\boldsymbol{v}}\|_{4, \partial \Omega}\|\boldsymbol{v}-\hat{\boldsymbol{v}}\|_{4, \partial \Omega} \\
& \leq M_{2}\|\hat{\boldsymbol{u}}-\boldsymbol{u}\|_{\partial \Omega}\|\hat{\boldsymbol{v}}\|_{4, \partial \Omega}\|\boldsymbol{v}-\hat{\boldsymbol{v}}\|_{4, \partial \Omega} \\
& \leq C(\Omega) M_{2} R\|\hat{\boldsymbol{u}}-\boldsymbol{u}\|_{1,2}\|\boldsymbol{v}-\hat{\boldsymbol{v}}\|_{1,2}
\end{aligned}
$$


and thus

$$
\|\boldsymbol{v}-\hat{\boldsymbol{v}}\|_{1,2} \leq C(\Omega) M_{2} K^{-2}\|\boldsymbol{h}\|_{1,2}\|\hat{\boldsymbol{u}}-\boldsymbol{u}\|_{1,2} .
$$

Hence, if $\|\boldsymbol{h}\|_{1,2}<K^{2} /\left(C(\Omega) M_{2}\right)$ then $\Phi$ is a contraction on $B(V, R)$, which is closed in $H^{1}(\Omega)^{3}$ and thus a complete metric space. Thus, by Banach's fixed point theorem, $\Phi$ has a unique fixed point, $\boldsymbol{v} \in B(V, R)$.

Remark 2. The restrictions on $F$ can be relaxed. It is sufficient to assume that $F(\cdot, \boldsymbol{u})$ is measurable (in stead of continuous) and that inequalities (3) and (4) hold for almost every $x \in \partial \Omega$. Then Lemma 2 and its proof must be adapted accordingly. However, this has no obvious advantage for modelling purposes. If condition (12) is the effective slip condition derived by an averaging procedure, one expects that $F$ will be at least continuous.

Proof of Lemma 1 By Theorem III.3.1 of Duvaut and Lions [23], there exists $\kappa=\kappa(\Omega)>0$ such that for all $\boldsymbol{v} \in H^{1}(\Omega)^{3}$,

$$
\|\mathbb{D}(\boldsymbol{v})\|^{2}+\|\boldsymbol{v}\|^{2} \geq \kappa\|\boldsymbol{v}\|_{1,2}^{2} .
$$

(In [23] it assumed that $\partial \Omega$ is Lipschitz continuous. Gobert [24] gives a different proof, which requires only that $\Omega$ has the cone property.) Furthermore, by using inequality (23), the compactness of the imbedding of $H^{1}(\Omega)$ into $L^{2}(\Omega)$, and a characterization of the set $\left\{\boldsymbol{v} \in H^{1}(\Omega)^{3}: \mathbb{D}(\boldsymbol{v})=0\right.$ in $\left.\Omega\right\}$, one can prove by contradiction that there exists $\lambda=\lambda(\Omega, \Gamma)>0$ such that for all $\boldsymbol{v} \in H^{1}(\Omega)^{3}$,

$$
\|\mathbb{D}(\boldsymbol{v})\|^{2}+\|\boldsymbol{v}\|_{\Gamma}^{2} \geq \lambda\|\boldsymbol{v}\|^{2} .
$$

(Hlaváček and Nečas $[25,26]$ use this approach in a more general setting to derive several Korn type inequalities.) Thus, for all $A, B>0$ and all $\boldsymbol{v} \in H^{1}(\Omega)^{3}$,

$$
\begin{aligned}
A\|\mathbb{D}(\boldsymbol{v})\|^{2}+B\|\boldsymbol{v}\|_{\Gamma}^{2} \\
\quad=\frac{A \lambda}{\lambda+1}\|\mathbb{D}(\boldsymbol{v})\|^{2}+\frac{A}{\lambda+1}\|\mathbb{D}(\boldsymbol{v})\|^{2}+B\|\boldsymbol{v}\|_{\Gamma}^{2} \\
\quad \geq \min \left\{(\lambda+1)^{-1} A, B\right\}\left(\lambda\|\mathbb{D}(\boldsymbol{v})\|^{2}+\|\mathbb{D}(\boldsymbol{v})\|^{2}+\|\boldsymbol{v}\|_{\Gamma}^{2}\right) \\
\quad \geq \kappa \lambda \min \left\{(\lambda+1)^{-1} A, B\right\}\|\boldsymbol{v}\|_{1,2}^{2}
\end{aligned}
$$

by inequalities (24) and (23).

Remark 3. (a) Inequality (17) is not in the usual form of a Korn type inequality but it is slightly stronger than

$$
A\|\mathbb{D}(\boldsymbol{v})\|^{2}+B\|\boldsymbol{v}\|_{\Gamma}^{2} \geq \frac{\kappa \lambda}{\lambda+1} \min \{A, B\}\|\boldsymbol{v}\|_{1,2}^{2},
$$

which follows from

$$
\|\mathbb{D}(\boldsymbol{v})\|^{2}+\|\boldsymbol{v}\|_{\Gamma}^{2} \geq \frac{\kappa \lambda}{\lambda+1}\|\boldsymbol{v}\|_{1,2}^{2}, \boldsymbol{v} \in H^{1}(\Omega)^{3} .
$$

(b) It follows from Lemma 1 and the proof of Theorem 1 that it is sufficient for Theorem 1 that $F(x, \cdot) \geq F_{L}>0$ on some $\Gamma \subset \partial \Omega$ with $|\Gamma|>0$. Thus one could apply the condition of perfect slip on a part of $\partial \Omega \backslash \Gamma$. 
Proof of Lemma 2 Inequality (18) follows from inequalities (4) and the definitions of $F_{a}$ and $\widetilde{G}$. Now consider inequality (19). Let $(x, \boldsymbol{v}),(x, \boldsymbol{w}) \in$ $T(\partial \Omega)$. Suppose that $|\boldsymbol{v}| \leq r_{0}$. If $|\boldsymbol{w}| \leq r_{0}$, then

$$
|\widetilde{G}(x, \boldsymbol{v})-\widetilde{G}(x, \boldsymbol{w})|=\left|F_{a}(x)-F_{a}(x)\right|=0 .
$$

If $r_{0}<|\boldsymbol{w}|<r_{1}$, then, by the mean value theorem and inequalities (4),

$$
\begin{aligned}
|\widetilde{G}(x, \boldsymbol{v})-\widetilde{G}(x, \boldsymbol{w})| & =\left|\left(\varphi\left(r_{0}\right)-\varphi(|\boldsymbol{w}|)\right)\left(G(x, \boldsymbol{w})-F_{a}(x)\right)\right| \\
& \leq\left(F_{U}-F_{L}\right) M_{1}\left|r_{0}-\right| \boldsymbol{w}|| \\
& \leq\left(F_{U}-F_{L}\right) M_{1}|| \boldsymbol{v}|-| \boldsymbol{w}|| \\
& \leq\left(F_{U}-F_{L}\right) M_{1}|\boldsymbol{v}-\boldsymbol{w}| .
\end{aligned}
$$

Similarly, if $|\boldsymbol{w}| \geq r_{1}$, then

$$
\begin{aligned}
|\widetilde{G}(x, \boldsymbol{v})-\widetilde{G}(x, \boldsymbol{w})| & =\left|\left(\varphi\left(r_{0}\right)-\varphi\left(r_{1}\right)\right)\left(G(x, \boldsymbol{w})-F_{a}(x)\right)\right| \\
& \leq\left(F_{U}-F_{L}\right) M_{1}\left|r_{0}-r_{1}\right| \\
& \leq\left(F_{U}-F_{L}\right) M_{1}|\boldsymbol{v}-\boldsymbol{w}| .
\end{aligned}
$$

Now suppose that $r_{0}<|\boldsymbol{v}|<r_{1}$. If $r_{0}<|\boldsymbol{w}|<r_{1}$, then

$$
\begin{aligned}
& |\widetilde{G}(x, \boldsymbol{v})-\widetilde{G}(x, \boldsymbol{w})| \\
& \quad=\left|\varphi(|\boldsymbol{v}|)\left(G(x, \boldsymbol{v})-F_{a}(x)\right)-\varphi(|\boldsymbol{w}|)\left(G(x, \boldsymbol{w})-F_{a}(x)\right)\right| \leq A+B,
\end{aligned}
$$

where

$$
A=\left|(\varphi(|\boldsymbol{v}|)-\varphi(|\boldsymbol{w}|))\left(G(x, \boldsymbol{v})-F_{a}(x)\right)\right| \leq\left(F_{U}-F_{L}\right) M_{1}|\boldsymbol{v}-\boldsymbol{w}|
$$

and, by inequality (3),

$$
\begin{aligned}
B & =|\varphi(|\boldsymbol{w}|)(G(x, \boldsymbol{v})-G(x, \boldsymbol{w}))| \\
& \leq\left. M_{0}|| \boldsymbol{v}\right|^{-1} \boldsymbol{v}-|\boldsymbol{w}|^{-1} \boldsymbol{w} \mid \\
& =M_{0}|\boldsymbol{v}|^{-1}\left|\boldsymbol{v}-\boldsymbol{w}+\left(1-|\boldsymbol{w}|^{-1}|\boldsymbol{v}|\right) \boldsymbol{w}\right| \\
& \leq 2 M_{0}\left(r_{0}\right)^{-1}|\boldsymbol{v}-\boldsymbol{w}|
\end{aligned}
$$

Similarly, if $|\boldsymbol{w}| \geq r_{1}$, then one proceeds as in the preceding case with $\varphi\left(r_{1}\right)$ in place of $\varphi(|\boldsymbol{w}|)$ and uses || $\boldsymbol{v}\left|-r_{1}\right| \leq|| \boldsymbol{v}|-| \boldsymbol{w}||$ to obtain the same estimates of $A$ and $B$.

Lastly, suppose that $|\boldsymbol{v}| \geq r_{1}$ and $|\boldsymbol{w}| \geq r_{1}$. Then, similar to the estimate of $B$ above,

$$
|\widetilde{G}(x, \boldsymbol{v})-\widetilde{G}(x, \boldsymbol{w})|=|G(x, \boldsymbol{v})-G(x, \boldsymbol{w})| \leq 2 M_{0}\left(r_{1}\right)^{-1}|\boldsymbol{v}-\boldsymbol{w}| .
$$

Hence, inequality (19) holds with $M_{2}=2 M_{0}\left(r_{0}\right)^{-1}+\left(F_{U}-F_{L}\right) M_{1}$. 


\section{References}

[1] E. Charrault T. Lee and C. Neto. Interfacial slip on rough, patterned and soft surfaces: A review of experiments and simulations. Adv. Colloid Interface Sci., 210:21-38, 2014.

[2] C. L. M. H. Navier. Mémoire sur les lois du mouvement des fluides. Mem. Acad. R. Sci. Inst. Fr., 6:191-242, 1823.

[3] M. J. Miksis and S. H. Davis. Slip over rough and coated surfaces. J. Fluid Mech., 273:125-139, 1994.

[4] Y. Achdou, P. Le Tallec, F. Valentin, and O. Pironneau. Constructing wall laws with domain decomposition or asymptotic expansion. Comput. Methods Appl. Mech. Engrg., 151:215-232, 1998.

[5] Y. Achdou, O. Pironneau, and F. Valentin. Effective boundary conditions for laminar flows over periodic rough boundaries. J. Comput. Phys., 147:187-218, 1998.

[6] W. Jäger and A. Mikelić. On the roughness-induced effective boundary conditions for an incompressible viscous flow. J. Differential Equations, 170:96-122, 2001.

[7] E. Marušić-Paloka. Average of Navier's law on the rapidly oscillating boundary. J. Math. Anal. Appl., 259:685-701, 2001.

[8] A. Mikelić. Recent developments in multiscale problems coming from fluid mechanics. In M. Kirkilionis et al., editor, Trends in Nonlinear Analysis, pages 225-267. Springer, Berlin, 2003.

[9] D. Bresch, F. Guillén-González, N. Masmoudi, and M. A. RodríguezBellido. Asymptotic derivation of a Navier condition for the primitive equations. Asymptot. Anal., 33:237-259, 2003.

[10] D. Bucur, E. Feireisl, and Š. Nečasová. On the asymptotic limit of flows past a ribbed boundary. J. Math. Fluid Mech., 10:554-568, 2008.

[11] A. Mikelić, Š. Nečasová, and M. Neuss-Radu. Effective slip law for general viscous flows over an oscillating surface. Math. Methods Appl. Sci., 36:20862100, 2013.

[12] Y. Amirat, O. Bodart, U. De Maio, and A. Gaudiello. Effective boundary condition for stokes flow over a very rough surface. J. Differential Equations, 254:3395-3430, 2013.

[13] D. Gérard-Varet. The Navier wall law at a boundary with random roughness. Comm. Math. Phys., 286:81-110, 2009.

[14] D. Gérard-Varet and N. Masmoudi. Relevance of the slip condition for fluid flows near an irregular boundary. Comm. Math. Phys., 295:99-137, 2010. 
[15] A.-L. Dalibard and D. Gérard-Varet. Effective boundary condition at a rough surface starting from a slip condition. J. Differential Equations, 251:3450-3487, 2011.

[16] M. Bonnivard, F.J. Suárez-Grau, and G. Tierra. On the influence of wavy riblets on the slip behaviour of viscous fluids. Z. Angew. Math. Phys., 67:27, 2016.

[17] C.-O. Ng and C. Y. Wang. Effective slip for Stokes flow over a surface patterned with two- or three-dimensional protrusions. Fluid Dyn. Res., 42:065504, 2011.

[18] C. Y. Wang. Stagnation flow on a plate with anisotropic slip. Eur. J. Mech. B Fluids, 38:73-77, 2013.

[19] A. T. Tran, H. Le Quang, and Q.-C. He. Effective interfacial conditions for the Stokes flow of a fluid on periodically rough surfaces. Acta Mech., 228:1851-1869, 2017.

[20] R. A. Adams. Sobolev Spaces, volume 65 of Pure Appl. Math. Academic Press, New York, 1975.

[21] P. Lax and N. Milgram. Parabolic equations. In Contributions to the Theory of Partial Differential Equations, volume 33 of Ann. of Math. Stud., pages 167-190. Princeton University Press, Princeton, 2003.

[22] G. P. Galdi. An Introduction the Mathematical Theory of the Navier-Stokes Equations. Springer Monogr. Math. Springer, New York, 2nd edition, 2011.

[23] G. Duvaut and J. L. Lions. Inequalities in Mechanics and Physics, volume 219 of Grundlehren Math. Wiss. Springer Verlag, Berlin, 1976.

[24] J. Gobert. Une inéquation fondamentele de la théorie de l'élasticité. Bull. Soc. Roy. Sci. Liège, 31:182-191, 1962.

[25] I. Hlaváček and J. Nečas. On inequalities of Korn's type. I. Boundary-value problems of elliptic systems of partial differential equations. Arch. Ration. Mech. Anal., 36:305-311, 1970.

[26] I. Hlaváček and J. Nečas. On inequalities of Korn's type. II. Applications to linear elasticity. Arch. Ration. Mech. Anal., 36:312-334, 1970. 